\title{
MANEJO PSICOTERAPÊUTICO DE TENTATIVAS DE SUICÍDIO EM ADOLESCENTES COM TRANSTORNO DE DÉFICIT DE ATENÇÃO E HIPERATIVIDADE (TDAH)
}

\section{ARTIGO ORIGINAL}

ROBERTO, Tiago Moreno Lopes ${ }^{1}$

ARAÚJO FILHO, Gerardo Maria de ${ }^{2}$

SILVA, Ligia Maria da ${ }^{3}$

ROBERTO, Tiago Moreno Lopes. ARAÚJO FILHO, Gerardo Maria de. SILVA, Ligia Maria da. Manejo psicoterapêutico de tentativas de suicídio em adolescentes com Transtorno de Déficit de Atenção e Hiperatividade (TDAH). Revista Científica Multidisciplinar Núcleo do Conhecimento. Ano 05, Ed. 10, Vol. 16, pp. 149-165. Outubro de 2020. ISSN: 2448-0959, Link de acesso: https://www.nucleodoconhecimento.com.br/psicologia/manejopsicoterapeutico

\section{RESUMO}

Trata-se de três casos de adolescentes com idade entre 14 e 17 anos, sendo dois do sexo feminino e um do sexo masculino, diagnosticados com Transtorno do déficit de atenção com hiperatividade - TDAH, encaminhados para Psicoterapia clínica na abordagem comportamental devido os pensamentos suicidas e tentativas. $\mathrm{O}$ foco delimitado consistiu em trabalhar as características depressivas do paciente e diminuindo aos comportamentos impulsivos de autolesão. A análise dos casos

\footnotetext{
${ }^{1}$ Graduado em Psicologia - UNIFEV - Mestre em Psicologia e Saúde - FAMERP.

2 Médico Psiquiatra; Mestre e Doutor em Neurociências - UNIFESP; Pós-doutor em Psiquiatria pela UNIFESP.

${ }^{3}$ Graduada em Psicologia - UNORP - Mestre em Psicologia e Saúde - FAMERP.
} 
demonstrou similaridades nas vivências na fase da infância, fatores traumáticos na área da sexualidade, abuso sexual nos três casos, gerando um distanciamento das habilidades socioafetivas na vida adulta. A peculiaridade dos casos demonstrou necessidade de mudanças nos padrões sociais e rotinas, onde o foco terapêutico era direcionado ao adolescente com intervenções em conjunto com os familiares.

Palavras-chave: Psicoterapia comportamental, TDAH, lesão auto provocada.

\section{INTRODUÇÃO}

O Transtorno de Déficit de Atenção e Hiperatividade (TDAH), tem sido objeto de diversas investigações científicas, atravessado inúmeras controvérsias em torno de seus determinantes e sua prevalência, em razão dos critérios que cada país utiliza na realização das pesquisas, suas abordagens diagnósticas e a fonte dessas informações. $O$ crescente diagnóstico do transtorno em crianças e adolescentes, com indicação para tratamento medicamentoso, tem ganhado atenção das produções científicas (POLANCZYK et al., 2014; FERREIRA; MOSCHETA, 2019).

Pesquisas apontam uma prevalência mundial de TDAH em crianças e adolescentes em cerca de 5,29\% (POLANCZYK et al., 2007). De acordo com o DSM V, o TDAH é classificado na categoria dos distúrbios do neurodesenvolvimento e estima-se que $5 \%$ das crianças e 2,5\% dos adultos possuem o transtorno. Esta patologia está frequentemente presente em crianças com idade entre 6 a 12 anos (ROHDE; DORNELES; COSTA, 2006). Há uma maior incidência em meninos do que em meninas e se estende para a vida adulta com uma proporção de cerca de 2:1 nas crianças e de 1,6:1 nos adultos (APA, 2013).

Pessoas com diagnóstico de TDAH, apresentam sintomas que acarretam prejuízos no funcionamento social, acadêmico e profissional. A principal característica do transtorno é um padrão persistente de desatenção e/ou hiperatividade-impulsividade (APA, 2013; BONADIO; MORI, 2013). Os comportamentos desatentos são observados por meio de divagação em tarefas, falta de persistência, dificuldade de manter o foco e desorganização, podendo ser primeiramente manifestado por 
pessoas do sexo feminino. A hiperatividade é externalizada pelo excesso da atividade motora e a impulsividade por comportamentos precipitados de alto prejuízo ao indivíduo. Os sintomas hiperativos são menos observáveis na adolescência e na vida adulta. No entanto, as consequências do TDAH podem persistir até a vida adulta. Embora exista a estabilização do transtorno no início da adolescência, alguns apresentam piora do quadro, manifestando comportamentos antissociais. Importante considerar que as manifestações deste adoecimento devem ocorrer em mais de um ambiente onde a pessoa está inserida (APA, 2013).

Estudos demonstram que independente da persistência do transtorno, os sintomas de desatenção que comprometem o desempenho escolar do indivíduo na infância permanecem durante a adolescência (DORNELES et al., 2013).

O desenvolvimento neurológico da criança, por meio da plasticidade das conexões nervosas, fornece oportunidades que contribuem no processo de aprendizagem mediante novas experiências. Por isso, quanto antes ela for diagnosticada, maior as possibilidades de novas ações, favorecendo este desenvolvimento (ROTTA; OHLWEILER; RIESGO, 2006).

Para Polanczyk e colaboradores (2007), o TDAH é um transtorno neurobiológico, genético, integrando também fatores ambientais. Segundo Barkley (2009), com base nos estudos realizados ao longo da história acerca desse transtorno, sua caracterização vem sendo apresentada com aspecto cada vez mais biológico e genético. Um dos fatores é a compreensão de que há um déficit de autocontrole e inibição de respostas das habilidades cognitivas, impactando diretamente no desenvolvimento da moral. Desta forma, o uso da medicação estimulante vem sendo defendida para o tratamento do TDAH, pois atuam controlando os efeitos do transtorno nas regiões cerebrais, diminuindo os sintomas que são provocados por base genética, desconsiderando quaisquer outros fatores históricos e culturais envolvidos no desenvolvimento dos comportamentos gerados pelo déficit (ROTHENBERGER; BANASCHEWSKI, 2007). 
O diagnóstico de TDAH também é apresentado decorrente da combinação de fatores ambientais e clínicos, sendo sua base neurológica. $O$ transtorno pode sofrer influência de fatores genéticos, estando presente em $25 \%$ dos parentes de primeiro grau (ROTTA; BRIDI FILHO; BRIDI, 2016).

Segundo Curatolo; D’Agati e Moavero (2010), limitar a compreensão do TDAH a uma única causa é desconsiderar a complexidade do transtorno, que possui característica crônica com impacto nas regiões do cérebro responsáveis por autorregulação, controle dos impulsos e percepção, no desenvolvimento e comportamento do indivíduo.

Muito mais que considerar o aspecto biológico do transtorno, há a necessidade de refletir sobre os diversos fatores que permeiam a adaptação das crianças aos padrões de comportamentos socialmente definidos. As demandas e as necessidades de adaptação da sociedade contemporânea têm sido cada vez mais complexas. No entanto, medicar, é o meio de controle social mais rápido, abstendo os responsáveis pelo processo de diagnóstico, a família e outros envolvidos na dinâmica com a criança, a refletir sobre sua responsabilidade (GIRÃO; COLAÇO, 2018).

Estudo realizado por Graeff e Vaz (2008), descreve que o diagnóstico deve ser feito por equipe multiprofissional, valendo-se de diversos recursos como escalas, testes psicológicos e neuropsicológicos. Apesar de contar com diversos profissionais, há bastante dificuldade para chegar a uma conclusão sobre o déficit.

Atualmente, a conduta mais adotada e considerada suficiente para o diagnóstico é a análise clínica, realizada por meio de observação dos critérios apresentados pelo DSM (GIRÃO; COLAÇO, 2018; FERREIRA; MOSCHETA, 2019).

Os impactos de ordem psicossociais para o indivíduo com TDAH são diversos. Estudos de acompanhamento realizados com criança diagnosticadas com o déficit, quando comparadas ao grupo controle, evidenciam maior comprometimento do indivíduo na esfera acadêmica, demonstrando menor desempenho escolar, suspensão e expulsão da escola, maior inserção em programas para o 
desenvolvimento das necessidades educacionais e menor taxa de egresso na universidade com baixo índice de formação. Quando adultos, na carreira profissional, os índices de demissões são maiores, com baixo desempenho nas atividades e maior frequência na mudança de emprego. A atividade de dirigir também é afetada. Adultos com TDAH são menos prudentes na condução de veículos com maior incidência de acidentes e multas de trânsito (BARKLEY, 2002).

Importante salientar, que embora as medicações sejam os mais indicados para o tratamento do TDAH, elas não atuam no desenvolvimento de habilidades sociais (THIENEMANN, 2005). O tratamento deve combinar a intervenção medicamentosa, responsável pela redução dos principais sintomas, que atuará no organismo sobre a predisposição de agir de maneira impulsiva ou agressiva e o acompanhamento psicológico, para reforçar mudanças comportamentais, estimulando hábitos mais saudáveis (MONGIA; HECHTMAN, 2016; SPRICH et al., 2016) e, que dependendo da gravidade do déficit e estado do paciente, a terapia comportamental pode ser indicada sem associação medicamentosa (AAP, 2011).

A literatura ainda ressalta um conjunto de intervenções para atuar sobre as questões comportamentais do indivíduo nos diversos contextos, como psicoeducar o paciente e seus familiares em relação ao transtorno e às comorbidades (CORDIOLI; GALLOIS; ISOLAN, 2015), treino parental no manejo de habilidades sociais, intervenções que podem acontecer dentro da sala de aula, manejo de contingências, dentre outras ações que podem contribuir de maneira significativa no tratamento (BARKLEY, 2009; CHRONIS et al., 2004; WEISS; WEISS, 2002).

O tratamento padronizado é a combinação de intervenções comportamentais que poderiam envolvera psicoeducação, treino parental no manejo de habilidades sociais, intervenções feitas na sala de aula, manejo de contingências, treino de habilidades sociais, dentre outras; além de medicamentos estimulantes, sendo que, em alguns casos, a depender da gravidade, a terapia comportamental poderia ser recomendada sem medicamentos (AAP, 2011; BARKLEY, 2006; CHRONIS et al., 2004; WEISS; WEISS, 2002). 
As pesquisas mostram que pacientes com TDAH apresentam vários transtornos comórbidos, sendo fundamental avaliá-los para maior efetividade do tratamento. Para Cordioli; Gallois e Isolan (2015), as principais comorbidades na infância relacionamse ao Transtorno de aprendizagem (10 a 50\%), Transtorno Opositor Desafiante (aproximadamente 60\%), Transtorno de Conduta (entre 30 a 50\%) e Transtorno de Ansiedade (20 a 25\%). De acordo com o DSM V, transtorno depressivo maior também é comórbido com TDAH. Nos adultos, podem ser comórbidos o transtorno explosivo intermitente, transtorno da personalidade antissocial e outros transtornos da personalidade. Além disso, apesar dos transtornos por abuso de substância ser mais comumente entre adultos com TDAH (9 a 40\%), diversos estudos mostram alta prevalência da comorbidade entre o déficit e abuso ou dependência de drogas na adolescência. As comorbidades associadas, exercem um efeito negativo e grande risco para a pessoa, sendo possível que este abuso ou dependência de drogas seja mais frequente em adolescentes com o diagnóstico de TDAH com associação do transtorno de conduta (ROHDE; DORNELES; COSTA, 2006).

Considerando os grandes riscos existentes pela combinação das comorbidades, a comunidade científica manifesta uma importante preocupação com a associação entre TDAH, ideação, tentativas e comportamento suicida. Alguns estudos denotam que esta associação pode ser um grande risco para comportamentos suicidas de crianças e adultos, porém, são necessárias maiores evidências sobre esta relação direta (AGOSTI; CHEN; LEVIN, 2011; TAYLOR; BODEN; RUCKLIDGE, 2014).

A Organização Mundial da Saúde (OMS) relata o suicídio como um dos maiores problemas de saúde pública, destacando-se entre as principais causas de morte em crianças de 5 a 14 anos e jovens de 15 a 29 anos (WHO, 2014).

Um estudo relata o crescimento no risco de suicídio por idade na América, sendo a maior taxa 16,69 entre adultos de 40 a 49 anos. A crescente taxa anual demonstrada entre crianças e adolescentes também possui considerável relevância. A taxa entre crianças de 5 a 9 anos passa de 0,03 para 100.000 e entre as crianças de 10 a 14 anos o índice é de 1,29. Entre adolescentes de 15 a 19 anos a taxa é de 7,53 e entre adultos jovens de 20 a 29 anos, 13,92 (HAUSER; GALLING; CORRELL, 2013). 
Há um grande e crescente risco de suicídio entre pacientes jovens diagnosticados com TDAH, evidenciado em pesquisas observacionais (MANOR et al. 2010; MCCARTHY et al., 2009). Estudos descrevem este risco maior em adolescentes e jovens do sexo masculino (KELLY; CORNELIUS JR; CLARK, 2004; JAMES; LAI; DAHL, 2004; LAM, 2002; MILLER; CHILES; BARNES, 1982), além da presença de comorbidades que frequentemente coocorre com o TDAH, sendo relevante seu fator de risco para o comportamento suicida, destacando-se os distúrbios de comportamento e depressão (JAMES; LAI; DAHL, 2004).

\section{RELATO DO CASO}

\section{IDENTIFICAÇÃO E DESCRIÇÃO GERAL DO PACIENTE}

Ana (os nomes são fictícios), 15 anos, branca, brasileira, solteira, cursava ensino fundamental. A paciente tinha estatura e peso médios e, geralmente, se vestia sempre com roupas largas, com higiene e cuidados básicos prejudicados.

Gabriel (os nomes são fictícios), 17 anos, branco, brasileiro, solteiro, iniciava o ensino superior. O paciente tinha estatura alta e peso um pouco acima, geralmente se vestia com roupas mais formais.

Monica (os nomes são fictícios), 16 anos, branca, brasileira, solteira, cursava ensino médio. A paciente tinha estatura alta e magra, geralmente se vestia com roupas claras e cuidados básicos prejudicados.

\section{IMPRESSÕES INICIAIS}

Nas primeiras sessões, Ana e Monica vieram acompanhadas de seus familiares, já o Gabriel não veio acompanhado de seus familiares, pois residiam em outra cidade, porém o agendamento foi realizado pela sua mãe. Todos os casos separadamente vieram com encaminhamento do médico neurologista, apresentando inicialmente sinais de dificuldades de aprendizado, angústia, choro fácil e pensamentos autodestrutivos. 
As duas pacientes do sexo feminino solicitaram aos familiares que saíssem da sala durante o atendimento, após os familiares relatarem alguns eventos antecedentes. Notou-se maior naturalidade ao expressarem sozinhas, ficando claro que a relação familiar era um impedimento para iniciar algumas temáticas intimas, onde Ana solicita para não retransmitir à sua família, e pede para garantir o sigilo do atendimento.

Nesse momento da sessão, o Psicoterapeuta afirma e garante as questões éticas de sigilo profissional e ressalta que quando necessário será chamado os familiares juntamente com a adolescente atendida; sendo assim nota-se que o vínculo e o acordo feito são fundamentais na busca pela confiança nas primeiras sessões.

A busca incessante dos familiares para saber o que era dito/conversado durante 0 atendimento era constante, porém foi ressaltado aos familiares a importância do vínculo terapêutico e as questões que poderiam dificultar a aproximação e abertura do adolescente.

\section{MOTIVO DA CONSULTA E SINTOMAS APRESENTADOS}

O motivo pela busca da psicoterapia havia suas particularidades entre os casos, mas o motivo de maior relevância e preocupação para os familiares de todos adolescentes eram as queixas de tristeza; autolesão e pensamentos de morte; bem como dificuldades de sociabilização e de concentração no âmbito educacional, sendo que todos os casos haviam diagnóstico laudo de Transtorno do Déficit de Atenção com Hiperatividade (TDAH).

Os comportamentos de autolesão deram início em momentos de enfrentamentos e dificuldades no âmbito escolar, os casos apresentavam níveis de dificuldades diferentes, prejuízos graves nas habilidades sociais e de aprendizagem, apresentando comportamentos dissociativos, principalmente no início da vida sexual, destoando-se da fase dos demais alunos de sua convivência; comportamentos que eram vistos pelos professores como "vulgares" ou falta de bons modos. Essa visão de julgamento se estendia entre amigos da escola no contexto familiar. 
O desejo pelo início do ato sexual foi detectado nos primeiros atendimentos; mentiras e vantagens eram expressamente ditas no momento em que o paciente relatava as intercorrências na escola e que demonstrava sua fragilidade e dificuldade ao lidar com seu desejo e com o seu próprio corpo. A sensibilidade do adolescente e os pedidos de desculpas incessantes evidenciavam o sofrimento e o sentimento de julgamento de si mesmo, o sentimento de culpa e de não pertencimento ao seu ambiente social de rotina.

\section{RESUMO DA HISTÓRIA PREGRESSA E ATUAL}

Ana era uma adolescente que passou por momentos traumáticos em sua infância, onde foram identificados sinais de abuso sexual na infância. Durante seu desenvolvimento, familiares notaram sua dificuldade de transmitir sentimentos, logo após vieram as primeiras lesões e tentativas de suicídio.

Ana apresentava idade intelectual distante de sua fase atual, apresentando muitas vezes irritabilidade e descontentamento por não pertencer aos grupos sociais de sua classe, onde passou por Bullying em sua escola.

Monica era uma adolescente com boa comunicação, porém com dificuldades de se desenvolver em relacionamentos afetivos, ficando muito cômodo sua aproximação com meios virtuais; os meios tecnológicos proporcionavam a ideia ou pensamento de pertencimento ao um grupo social, tornando-as reforçadoras e compulsivas, usando do ambiente virtual como principal ferramenta de socialização. Seu sentimento por não acompanhar um grupo social gerou momentos de isolamento em seu ambiente escolar, ocasionando a ideação suicida.

Em alguns momentos sentiu-se atraída por pessoas com as quais nunca tivera contato presencial, demonstrando que o meio virtual era uma das formas de reforçar suas dificuldades, alimentando uma falsa ilusão sobre seu real desejo.

Gabriel apresentava pouco contato familiar, suas vivências apresentavam sintomas depressivos e sinais de abuso sexual na infância, onde não conseguia realizar 
atividades de rotina, demonstrando desorganização para execução de atividades essenciais.

\section{HIPÓTESES DE TRABALHO}

1 Hipótese Diagnóstica: TDAH. Os critérios para esta patologia, de acordo com a quarta edição do Manual Diagnóstico e Estatístico dos Transtornos Mentais (APA, 1994;1995).

2 Hipóteses: a) dificuldade de se relacionar com seu meio social; b) Ausência de reforçadores sociais $\mathrm{c}$ ) dificuldade para lidar com os desejos sexuais d) Pensamentos suicidas e tentativas

3 Foco: trabalhar novos repertórios sociais.

4 Atendimentos propostos inicialmente: 20; prorrogação: 20; total:40.

\section{EVOLUÇÃO DO PACIENTE DURANTE O PROCESSO}

Um dos primeiros objetivos terapêuticos foi conscientizar a todos os atendidos que o sigilo e a ética profissional estavam resguardados, foram geradas reflexões sobre a necessidade de atendimento e os benefícios que ele poderia ter ao longo da vida. $\mathrm{O}$ vínculo terapêutico formando foi fortalecido a cada sessão, demonstrando maior confiança e envolvimento.

As frustrações geradas pelas dificuldades de se relacionar em diversos contextos foram trabalhadas com uma postura ativa de enfretamento, sempre reforçando pequenas mudanças e progressos no âmbito educacional.

Diversas pesquisas sobre a interação de crianças com TDAH com outros adultos e crianças fora do contexto familiar, com amigos e professores, demonstram que as ordens e repressão aumentam, mães e professorem tendem a reprimir e ter mais disciplina, da mesma forma quando a criança com TDAH entra em um novo grupo social de amizade, os demais integrantes passam a agir como "pequenas mães", 
reforçando um padrão de disciplina do portador de TDAH. (BENCZIK; CASELLA, 2015).

Desta forma nota-se que a criança hiperativa se irrita com maior facilidade, despertando o conceito de não pertencimento ao grupo, onde os julgamentos incessantes de colegas e professores reforçam o padrão de isolamento social do portador e a busca pela paz e pelo desejo de ser aceito é encontrado no silêncio da solidão.

As cooperações no envolvimento de ações sociais foram estimuladas inicialmente em cada contexto familiar dos casos analisados, contudo notou-se que um dos familiares acabou cedendo às regras de rotinas, ficando claro que a família transfere um modelo inadequado, diante das dificuldades que o adolescente com TDAH apresenta.

Pesquisa conclui que algumas relações familiares dos portadores de TDAH se caracterizam por conflitos, coerção, estresse e disciplina que pode ser mais frouxa. Grande parte dos conflitos é gerado pelo impacto dos familiares; o comportamento dos familiares e seus padrões disfuncionais podem também contribuir com essas interações problemáticas dos adolescentes (BENCZIK; CASELLA, 2015).

Foi identificado que em todos os casos o abuso sexual se fez presente na infância, gerando fatores traumáticos e ocasionando disfunções psicológicas e neurológicas no desenvolvimento, traumas que geraram dificuldades na sociabilização. No âmbito terapêutico foi desmitificado a visão sobre $o$ ato sexual, foi trabalhado os aspectos que geravam maior desejo, onde foi direcionado os comportamentos disfuncionais para um contexto mais adequado.

Estudos ressaltam e afirmam que a exposição ao estresse crônico, como é o caso do abuso sexual na infância, reflete um estado de medo, causando efeitos negativos ao neurodesenvolvimento. A exposição do adolescente resulta em diversos prejuízos cognitivos. (BORGES; DELL'AGLIO, 2008).

O reconhecimento do próprio corpo para o adolescente durante a psicoterapia gerou reflexões sobre como lidar com os impulsos sexuais e sua forma de enxergar o sexo, 
com o comportamento da masturbação e da importância da não exposição às situações que poderiam colocá-los em constrangimentos. Foi fundamental trabalhar a conscientização da privatização e autocuidado com o corpo, reflexões sobre vestimentas e educação sexual.

\section{TEMAS PRINCIPAIS DURANTE SESSÕES}

- Sessão: Sentimentos de desmotivação - abordagem motivacional por meio de reforçadores sociais identificados.

- Sessão: Dificuldades de aprendizagem - abordagem estratégica de novos hábitos comportamentais, associado à rotina dos familiares.

- Sessão: Insegurança e medo ao lidar com a sexualidade - abordagem desmistificando conceitos e pré-conceitos.

- Sessão: Sentimentos e lesões - abordagem sensibilizando e compreendendo os sintomas.

- Sessão: Enfretamento dos desejos sociais - foi trabalhada a aceitação dos reforçadores positivos sociais, lidando e desenvolvendo novos repertórios sociais por meio do verbalizar.

- Sessão: identificando meus desejos e sonhos - abordagem dinâmica sobre como me vejo e como gostaria de estar.

- Sessão: lidando com as limitações - Aprender a lidar com as dificuldades e imprevistos diários no âmbito escolar e afetivo.

- Sessão: Responsabilidades e deveres - Compreender de uma forma prática o significado de uma rotina e seus aspectos positivos para alcançar objetivos.

- Sessão: Enfretamento das dificuldades de aproximação com grupos sociais abordagem estratégica com os familiares.

\section{TÉRMINO DO PROCESSO}

A visão de Gabriel sobre a terapia foi se modificando com o decorrer das sessões e intervenções propostas. 
"Quando não venho aqui, sinto falta de conversar, você sabe que tem coisas que só falo aqui, hoje sinto que sou mais organizado com minhas coisas, consigo focar e fazer".

Gabriel notou melhora em seus sintomas, relatando que aprendeu a lidar melhor com seus comportamentos, observou melhoras no rendimento escolar e afetivo. Ficou clara sua dificuldade de focar em seus desejos e planos, os eventos traumáticos de sua infância não eram vistos ou interpretados como doença; sendo assim, avaliada a necessidade de dar continuidade ao tratamento medicamentoso.

A visão de Monica sobre a terapia foi que ela proporcionou ferramentas para alcançar seus planos e desejos.

"Estou muito feliz, consegui beijar pela primeira vez, estou me sentindo mais bonita, parece que existe alguém que goste de mim assim".

"De fato concordo que beijar e ter uma pessoa real é bem melhor que namorar a distância".

Monica passou a não se sentir insegura ao iniciar seus desejos, ao longo prazo se estendeu a novos grupos de amizades de ambos sexos; anteriormente o sentimento de inferioridade e distorção do seu próprio corpo era constante, gerando isolamento social e tristeza.

Ana, no final do tratamento, passou a ter uma nova postura em relação à sua rotina $e$ seus meios sociais.

"Sou feliz, consigo me comunicar e me expressar, antes acabava sendo rebelde talvez por não me sentir ouvida". 


\section{DISCUSSÃO}

\section{FATORES QUE CONTRIBUÍRAM PARA O RESULTADO SATISFATÓRIO}

Entre os fatores que contribuíram para o resultado final satisfatório do processo terapêutico: todos adolescentes apresentaram uma aliança de vínculo positivo.

O treino de habilidades sociais dentro do ambiente terapêutico proporcionou maior empatia com as relações sociais, as quais foram bastante trabalhadas com foco nos ambientes de maior acesso.

O enfretamento no ambiente natural tem como consequência que as respostas adquiridas e reforçadas na interação acabam se propagando para os demais ambientes, deixando o controle das contingências naturais. (MARCO; CALAIS, 2012).

Diversos estudos descrevem que os adolescentes que se sentiam mais sozinhos e tristes apresentam prevalências mais altas de planejamento suicida do que aqueles que não apresentam esses sentimentos.

$O$ ato de lidar com os próprios sentimentos e acontecimentos de rotina proporcionou a eficácia de menor frequência de tentativas de lesões autoprovocadas, desencadeando maior resiliência às frustrações.

\section{CONCLUSÕES}

Diante do relato dos casos, nota-se que os adolescentes com TDAH estão em uma fase de descobertas e muitas inabilidades e expectativas dos familiares, bem como os fatores traumáticos (abuso sexual) acarretaram algumas mudanças e atrasos na vida adulta, gerando fatores depressivos com tentativas de suicídios; as sensações por não saber lidar com o desejo de se aproximar das pessoas se fazem ambíguas, ao mesmo tempo querem estar numa relação afetiva, ao mesmo tempo há o medo de enfrentar as próprias dificuldades. 
A relação com o ato sexual de abuso na infância se reflete nitidamente nas relações e comportamentos inadequados, o atraso cognitivo se torna claro e nítido nos grupos sociais e suas escolhas, o desejo de conseguir se satisfazer se faz constante, principalmente no realizar $\mathrm{o}$ ato sexual; porém, existe o distanciamento das habilidades, tornando cada vez maior o isolamento social, onde nota-se o surgimento de comportamentos fisiológicos exacerbados e disfuncionais, como masturbação em contextos atípicos.

A análise desse caso vem contribuir e corroborar que é possível por meio da psicoterapia comportamental ter um manejo clínico eficaz na busca por um olhar mais amplo sobre as queixas sociais, colocando uma visão sem julgamentos moralistas em favor de um reconhecimento e pertencimento de sua própria história. A imparcialidade do Psicoterapeuta proporcionou maior abertura e vínculo terapêutico, em que julgamentos foram desconstruídos juntamente com os familiares.

As mudanças de rotina, que envolveram todos do núcleo familiar, reforçaram nos adolescentes atitudes positivas, como desenvolver, a longo prazo, significados para seu ambiente, hábitos e atividades simples, que proporcionaram maior desempenho escolar e interromperam os pensamentos de morte e tentativas de suicídio.

\section{REFERÊNCIAS}

AAP - American Academy of Pediatrics. ADHD: Clinical Practice Guideline for the Diagnosis, Evaluation, and Treatment of Attention-Deficit/ Hyperactivity Disorder in Children and Adolescents. Pediatrics, v. 128, n. 5, 1007-22, 2011.

AGOSTI, Vito; CHEN, Ying; LEVIN, Frances R. Does attention deficit hyperactivity disorder increase the risk of suicide attempts? J Affect Disord, v. 133, n. 3, p. 595-9, 2011.

APA - American Psychiatric Association. Diagnostic and statistical manual of mental disorders. 5 ed. Arlington, VA: Author DSM V, 2013. 
Diagnostic and statistical manual of mental disorders - DSM. 4. ed. Washington: American Psychiatric Press Inc, 1994.

APA - Associação Americana de Psiquiatria. Manual diagnóstico e estatístico de transtornos mentais-DSM. 4 ed. Tradução: Dayse Batista. Porto Alegre: Artes Médicas, 1995.

BARKLEY, Russel A. Major life activity and health outcomes associated with attentiondeficit/hyperactivity disorder. The Journal of Clinical Psychiatry, v. 63, n. 12, p. 10-15, 2002.

. Transtorno de déficit de atenção/hiperatividade: manual para diagnóstico e tratamento. 3. ed. Porto Alegre: Artmed, 2009.

BENCZIK, Edyleine Bellini Peroni; CASELLA, Erasmo Barbante. Compreendendo o impacto do TDAH na dinâmica familiar e as possibilidades de intervenção. Rev. psicopedag., v. 32, n. 97, p. 93-103, 2015.

BONADIO, Rosana Aparecida Albuquerque; MORI, Nerli Nonato Ribeiro. Transtorno de Déficit de Atenção/Hiperatividade diagnóstico e prática pedagógica. Maringá: Eduem, 2013.

BORGES, Jeane Lessinger; DELL'AGLIO, Débora Dalbosco. Relações entre abuso sexual na infância, transtorno de estresse pós-traumático (TEPT) e prejuízos cognitivos. Psicol. Estud., v. 13, n. 2, p. 371-379, jun., 2008.

CHRONIS, Andrea. M. et al. An Evaluation of the Summer Treatment Program for Children with Attention-Deficit/Hiperactivity Disorder Using a Treatment Withdrawal Desig. Behavior Therapy, v. 35, n. 3, p. 561-585, 2004.

CORDIOLI, Aristides Volpato.; GALLOIS, Carolina Benedetto; ISOLAN, Luciano. (Org.). Psicofármacos: consulta rápida. Porto Alegre: Artmed, 2015.

CURATOLO, Paulo; D'AGATI, Elisa; MOAVERO, Romina. The neurobiological basis of ADHD. Ital J Pediatr, v. 36, n. 1, p. 79, 2010. 
DORNELES, Beatriz Vargas Dorneles. et al. Impacto do DSM-5 no Diagnóstico de Transtornos de Aprendizagem em Crianças e Adolescentes com TDAH: Um Estudo de Prevalência. Psicologia: Reflexão e Crítica, v. 27, n. 4, p. 759-767, 2013.

FERREIRA, Rodrigo Ramires.; MOSCHETA, Murilo dos Santos. A Multiplicidade do TDAH nas diferentes versões produzidas pelas ciências no Brasil. Psic.: Teor. e Pesq., v. 35, e3539, p. 1-11, 2019.

GIRÃO, Marina Serejo; COLAÇO, Veriana de Fátima Rodrigues. TDAH na infância contemporânea: um olhar a partir da sociologia da infância e da psicologia histórico cultural. Pesqui. prát. Psicossociais, v. 13, n. 1, p. 1-13, jan.-abr., 2018.

GRAEFF, Rodrigo Linck; VAZ, Cícero E. Avaliação e diagnóstico do transtorno de déficit de atenção e hiperatividade (TDAH). Psicologia USP, v. 19, n. 3, p. 341-361, jul., set. 2008.

HAUSER, Marta; GALLING, Britta; CORRELL Christoph U. Suicidal ideation and suicide attempts in children and adolescents with bipolar disorder: a systematic review of prevalence and incidence rates, correlates, and targeted interventions. Bipolar Disord, v. 15, v. 5, p. 507-23, aug., 2013.

JAMES, Anthony; LAI, F. H.; DAHL C. Attention deficit hyperactivity disorder and suicide: a review of possible associations. Acta Psychiatr Scand, n. 110, p. 408-415, 2004.

KELLY, Thomas M.; CORNELIUS, Jack R; CLARK, Ducan B. Psychiatric disorders and attempted suicide among adolescents with substance use disorders. Drug Alcohol Depend, ano. 7, v. 73, n. 1, p. 87-97, jan. 2004.

LAM, Lawrence T. Attention deficit disorder and hospitalization due to injury among older adolescents in New South Wales, Australia. J Atten Disord, v. 6, n. 2, p. 277282, 2002. 
MANOR; Iris. et al. Possible association between attention deficit hyperactivity disorder and attempted suicide in adolescents - a pilot study. Eur Psychiatry, v. 25, n. 3, p. 146-150, 2010.

MARCO, Mariana Nunes da Costa; CALAIS, Sandra Leal. Acompanhante terapêutico: caracterização da prática profissional na perspectiva da análise do comportamento. Rev. Bras. de Ter. Comp. Cogn., v. XIV, n. 3, p. 4-33, 2012.

MCCARTHY, Suzanne. et al. Mortality associated with attention-deficit hyperactivity disorder (ADHD) drug treatment: a retrospective cohort study of children, adolescents and young adults using the general practice research database. Drug Saf, v. 32, n. 11, p. 1089-96, 2009.

MILLER, Michael L.; CHILES, John A.; BARNES, Valerie E. Suicide attempters within a delinquent population. Journal of Consulting and Clinical Psychology, v. 50, n. 4, p. 491-498, 1982.

MONGIA, Monica; HECHTMAN, Lily. Attention Deficit Hyperactivity Disorder across the lifespan: Review of literature on Cognitive Behavior Therapy. Curr Dev Disord Rep, v. 3, n. 1, p. 7-14, 2016.

POLANCZYK, Guilherme V. et al. ADHD prevalence estimates across three decades: an updated systematic review and meta-regression analysis. Int J Epidemiol., v. 43, n. 2, p. 434-442, 2014.

POLANCZYK, Guilherme V. et al. The worldwide prevalence of ADHD: A systematic review and metaregression analysis. Am J Psychiatry, v. 164, n. 6, p. 942-948, 2007.

RIESGO, Rudimar dos Santos. Transtorno da atenção: Comorbidades. In: ROTTA, Newra Tellechea; OHLWEILER, Lygia; RIESGO, Rudimar dos Santos. Transtornos de aprendizagem: Abordagem neurobiológica e multidisciplinar. 2. ed. Porto Alegre: Artes Médicas, 2006. p. 347-363. 
ROHDE, Luiz Augusto Paim; DORNELES, Beatriz Vargas; COSTA, Adriana Corrêa. Intervenções escolares no transtorno de déficit de atenção/ hiperatividade. In: ROTTA, Newra Tellechea; OHLWEILER, Lygia; RIESGO, Rudimar dos Santos. Transtornos de aprendizagem: Abordagem neurobiológica e multidisciplinar. 2. ed. Porto Alegre: Artes Médicas, 2006.

ROTHENBERGER, Aribert, BANASCHEWSKI, Tobias. Informing the ADHD Debate. Scientific American, v. 17, ed. 2s, p. 36-41, jun. 2007.

ROTTA, Newra Tellechea; BRIDI FILHO, César Augusto, BRIDI, Fabiane De Souza. (Org). Neurologia e aprendizagem: Uma abordagem multidisciplinar. Porto Alegre: Artmed, 2016.

ROTTA, Newra Tellechea; OHLWEILER, Lygia; RIESGO, Rudimar dos Santos. Transtornos de aprendizagem: Abordagem neurobiológica e multidisciplinar. 2. ed. Porto Alegre: Artes Médicas, 2006.

SPRICH, Susan. E. et al. A randomized controlled trial of cognitive behavioral therapy for ADHD in medication-treated adolescents. J Child Psychol Psychiatry, 57 (11): 1227-1228, nov., 2016.

TAYLOR, Mairin R.; BODEN, Joseph M.; RUCKLIDGE, Julia J. The relationship between ADHD symptomatology and self-harm, suicidal ideation, and suicidal behaviours in adults: a pilot study. Atten Defic Hyperact Disord, v. 6, n. 4, p. 303-12, dez., 2014

THIENEMANN, Margo L. Group Psychotherapy. In: SADOCK, Benjamin James; SADOCK, Virginia A.; RUIZ, Pedro. Kaplan \& Sadock's: Comprehensive Textbook of Psychiatry, Philadelphia: Lippincott Williams \& Wilkins, 2005. p. 3343-3351.

WEISS, Margaret D; WEISS, Gabrielle. Attention Deficit Hyperactivity Disorder. In: MARTIN, Andrés; BLOCH, Michael H.; VOLKMAR, Fred R. Lewis Children and Adolescent Psychiatry: A Comprehensive Textbook. Philadelphia: Lippincott Williams \& Wilkins, 2002. p. 645-647. 
WHO - World Health Organization. Preventing suicide: A global imperative. Luxembourg: WHO, 2014.

Enviado: Setembro, 2020.

Aprovado: Outubro, 2020. 